\title{
EXTENSION OF NEUTROSOPHICSEMI OPEN SETS
}

\section{P. JEYA PUVANESWARI ${ }^{1} \&$ K. BAGEERATHI ${ }^{2}$}

${ }^{1}$ Assistant Professor of Mathematics, Vivekananda College, Agasteeswaram, Tasmil Nadu, India

${ }^{2}$ Assistant Professor of Mathematics, Aditanar College of Arts and Science, Tiruchendur, Tamil Nadu, India

\section{ABSTRACT}

Neutrosophic concepts mostly utilized in vague collection of items. Already we extend feebly open sets, semi open sets and its properties to neutrosophic space. Many Researchers extend the concepts of generalized closure operator into neutrosophy theory. Focal point of this write-up is to initiate neutrosophic *semi open setsconcerningthe operator neutrosophic generalized closure.

Mathematics Subject Classification: 03E72, $18 B 30$.

KEYWORDS: Phrases: Neutrosophic Generalized Closure, Neutrosophicsemi Open*, Neutrosophicsemi Closed* \& Neutrosophic Topological Space

Received: Oct 01, 2020; Accepted: Oct 21, 2020; Published: May 20, 2021; Paper Id.: IJMCARJUN20216

\section{INTRODUCTION}

In 1998, Smarandache [11] introduced the set on three components involving the membership, indeterminacy and non-membership values belongs to $\rfloor 0^{-}, 1^{+}\lfloor$a nonstandard unit interval. He represents the above three components respectively as $T, I, F$.

Let $T, I, F$ be real standard or not standard subset of $\rfloor 0,1^{+}\lfloor$, with

$$
\begin{aligned}
& \sup _{-} T=t_{-} \sup , \text { inf } f_{-}=t_{-} \text {inf } \\
& \sup _{-} I=i_{-} \sup , \text { inf } f_{-}=i_{-} \text {inf } \\
& \sup _{-} F=f_{-} \sup , \text { inf } f_{-}=f_{-} \text {inf }
\end{aligned}
$$

$n-\sup =t_{-}$sup $+i_{-} \sup +f_{-} \sup$

$n-\inf =t_{-} \inf +i_{-} i n f+f_{\text {inf }}, T, I, F$ are called neutrosophic components.

The preliminary concepts required in our work please refer $[5, \mathbf{6}, \mathbf{1 2}]$. The rest of this article is assembled come after. In section 2, the abstraction of neutrosophicsemi open* setis introduced along with its belongings are investigated. Section 3 deals with some properties on neutrosophicsemi closed* sets.

\section{NEUTROSOPHICSEMI OPEN* SETS}

This segment deals with a new class of sets, namely neutrosophicsemi open*make use of the neutrosophic generalized closure operator.

Proposition 2.1.Neutrosophic generalized closure operator(is denoted by $\mathrm{Ngcl}$ ) operator is a Kuratowski closure operator onneutrosophic topological spaces. 
Hereafter, "Ngcl" operator is designated by $N c l *$.

Definition 2.2. Let $\left(\mathcal{U}, \mathfrak{I}_{N}{ }^{*}\right)$ be a neutrosophic topological space on $\mathcal{K}$ generated by $N c l^{*}$. That is $\mathfrak{I}_{N}{ }^{*}=\left\{A: N c l^{*}\left(A^{C}\right)=\right.$ $A^{C}, A$ is a neutrosophic subset of $\mathcal{X}$.

Theorem 2.3. $\mathfrak{\Im}_{N} \subseteq \mathfrak{I}_{N}{ }^{*}$ with equality if and only ifeachneutrosophic generalized closed set in $\left(\varkappa, \mathfrak{I}_{N}\right)$ is neutrosophic closed $\operatorname{set} \operatorname{in}\left(\varkappa, \widetilde{I}_{N}{ }^{*}\right)$.

Proof. Let $\mathcal{L}$ be a neutrosophic closed set.Since $\mathcal{L} \subseteq N c l^{*}(\mathcal{R}) \subseteq N c l(\mathcal{L})$ implies that $\mathcal{L}$ is neutrosophic closedin $\Im_{N}{ }^{*}$. Thus $\mathfrak{J}_{N} \subseteq \mathfrak{I}_{N}{ }^{*}$. Also let $\mathfrak{I}_{N}=\mathfrak{I}_{N}{ }^{*}$ and let $\mathcal{R}$ be neutrosophic generalized closed in $\mathfrak{\Im}_{N}$. Then $\mathcal{R}=N c l *(\mathcal{R})$. That implies $\mathcal{R}$ is neutrosophic closed in $\mathfrak{I}_{N}=\mathfrak{I}_{N}{ }^{*}$. Thus eachneutrosophic generalized closed set in $\left(X, \mathfrak{I}_{N}\right)$ is neutrosophic closed set in $\left(\mathcal{X}, \mathfrak{I}_{N}\right)$.

Conversely, let eachneutrosophic generalized closed set in $\left(\mathcal{\varkappa}, \widetilde{J}_{N}{ }^{*}\right)$ is neutrosophic closed set in $\left(\varkappa, \widetilde{J}_{N}\right)$. Then $N c l^{*}(\mathcal{L})=\operatorname{Ncl}(\mathcal{L})$. Hence $\mathfrak{I}_{N}=\mathfrak{I}_{N}{ }^{*}$.

Definition 2.4.A neutrosophic subset $G$ of a neutrosophic topological space $\left(\varkappa, \mathfrak{I}_{N}\right)$ is called a neutrosophicsemi open*setif there is aneutrosophic open set $U$ in $\varkappa$ such that $U \subseteq G \subseteq N c l *(U)$.

Theorem 2.5. A neutrosophic subset $G$ is neutrosophicsemi open* if and only if $G \subseteq N c l *(\operatorname{Nint}(G))$.

Proof: If $G$ is neutrosophicsemi open*, then there is aneutrosophic open set $U$ such that $U \subseteq G \subseteq N c l^{*}(U)$. Now $U \subseteq$ $G \operatorname{implies} U=\operatorname{Nint}(U) \subseteq \operatorname{Nint}(G) \Longrightarrow G \subseteq N c l^{*}(U) \subseteq N c l^{*}(\operatorname{Nint}(G))$.

Conversely, assume that $G \subseteq N c l^{*}(\operatorname{Nint}(G))$.Take $U=\operatorname{Nint}(G)$. Then $U$ is aneutrosophic open set in $X$ such that $U \subseteq G \subseteq N c l *(U)$. Therefore $G$ is neutrosophicsemi open*.

Example 2.6.Let $\mathcal{\varkappa}=\{\alpha, \beta, \delta\}$ and $\mathfrak{I}_{N}=\left\{0_{N}, \wp, \mathbb{Q}, 1_{N}\right\}$. Then $\left(\mathcal{H}, \mathfrak{I}_{N}\right)$ is a neutrosophic topological space. The neutrosophic closed sets are $\mathfrak{I}_{N}{ }^{C}=\left\{1_{N}, \mathcal{R}, S, 0_{N}\right\}$ where,

$$
\begin{gathered}
\wp=\left\langle x,\left(\frac{\alpha}{0.4}, \frac{\beta}{0.4}, \frac{\delta}{0.5}\right),\left(\frac{\alpha}{0.4}, \frac{\beta}{0.4}, \frac{\delta}{0.54}\right),\left(\frac{\alpha}{0.3}, \frac{\beta}{0.4}, \frac{\delta}{0.4}\right)>,\right. \\
\mathbb{Q}=\left\langle x,\left(\frac{\alpha}{0.4}, \frac{\beta}{0.5}, \frac{\delta}{0.6}\right),\left(\frac{\alpha}{0.4}, \frac{\beta}{0.5}, \frac{\delta}{0.6}\right),\left(\frac{\alpha}{0.2}, \frac{\beta}{0.3}, \frac{\delta}{0.3}\right)>,\right. \\
\mathcal{R}=\left\langle x,\left(\frac{\alpha}{0.6}, \frac{\beta}{0.6}, \frac{\delta}{0.5}\right),\left(\frac{\alpha}{0.6}, \frac{\beta}{0.6}, \frac{\delta}{0.5}\right),\left(\frac{\alpha}{0.7}, \frac{\beta}{0.6}, \frac{\delta}{0.7}\right)>,\right. \\
S=\left\langle x,\left(\frac{\alpha}{0.6}, \frac{\beta}{0.5}, \frac{\delta}{0.4}\right),\left(\frac{\alpha}{0.6}, \frac{\beta}{0.5}, \frac{n \delta}{0.4}\right),\left(\frac{\alpha}{0.8}, \frac{\beta}{0.8}, \frac{\delta}{0.7}\right)>,\right.
\end{gathered}
$$

Define $T=<x,\left(\frac{\alpha}{0.5}, \frac{\beta}{0.6}, \frac{\delta}{0.5}\right),\left(\frac{\alpha}{0.5}, \frac{\beta}{0.8}, \frac{\delta}{0.7}\right),\left(\frac{\alpha}{0}, \frac{\beta}{0.2}, \frac{\delta}{0.1}\right)>$.

$\operatorname{Then} \operatorname{Nint}(T)=<x,\left(\frac{\alpha}{0.4}, \frac{\beta}{0.4}, \frac{\delta}{0.5}\right),\left(\frac{\alpha}{0.4}, \frac{\beta}{0.4}, \frac{\delta}{0.54}\right),\left(\frac{\alpha}{0.3}, \frac{\beta}{0.4}, \frac{\delta}{0.4}\right)>\operatorname{alsoN} c 1^{*}(\operatorname{Nint}(T))=$ $<x,\left(\frac{\alpha}{0.5}, \frac{\beta}{0.6}, \frac{\delta}{0.7}\right),\left(\frac{\alpha}{0.5}, \frac{\beta}{0.8}, \frac{\delta}{0.6}\right),\left(\frac{\alpha}{0.7}, \frac{\beta}{0.2}, \frac{\delta}{0}\right)>$. It can be verified that $T \subseteq N C C^{*}(\operatorname{Nint}(T))$.Hence $T$ is aneutrosophicsemi open set*.

Theorem 2.7.Each open set is semi open in general topological spaces. But this result is also true in neutrosophic spaces, that is each neutrosophic open set is neutrosophic semi open*. 
Proof: Take $U$ isneutrosophic open in $\varkappa$. Then $\operatorname{Nint}(U)=U$. Therefore $U \subseteq N c l^{*}(U)=N c l *(\operatorname{Nint}(U))$. Hence by Theorem 2.5, $U$ is neutrosophicsemi open*.

Example 2.8.Let $\mathcal{X}=\{\eta, \vartheta\}$ and $\widetilde{I}_{N}=\left\{0_{N}, A, B, C, D, 1_{N}\right\}$. Their correspondingclosed sets $\operatorname{are} \widetilde{\Im}_{N}{ }^{C}=$ $\left\{1_{N}, F, G, H, I, 0_{N}\right\}$ where, $A=\left\langle x,\left(\frac{\eta}{0.2}, \frac{\vartheta}{0.9}\right),\left(\frac{\eta}{0.1}, \frac{\vartheta}{0.8}\right),\left(\frac{\eta}{0.4}, \frac{\vartheta}{0.3}\right)>B=\left\langle x,\left(\frac{\eta}{0.3}, \frac{\vartheta}{0.3}\right),\left(\frac{\eta}{0.7}, \frac{\vartheta}{0.2}\right),\left(\frac{\eta}{0.7}, \frac{\vartheta}{0.7}\right)>, C=\right.\right.$ $<x,\left(\frac{\eta}{0.3}, \frac{\vartheta}{0.9}\right),\left(\frac{\eta}{0.7}, \frac{\vartheta}{0.8}\right),\left(\frac{\eta}{0.4}, \frac{\vartheta}{0.3}\right)>$, $D=<x,\left(\frac{\eta}{0.2}, \frac{\vartheta}{0.3}\right),\left(\frac{\eta}{0.1}, \frac{\vartheta}{0.2}\right),\left(\frac{\eta}{0.7}, \frac{\vartheta}{0.7}\right)>, E=\left\langle x,\left(\frac{\eta}{0.8}, \frac{\vartheta}{0.1}\right),\left(\frac{\eta}{0.9}, \frac{\vartheta}{0.2}\right),,\left(\frac{\eta}{0.6}, \frac{\vartheta}{0.7}\right)>, F=\right.$
$<x,\left(\frac{\eta}{0.7}, \frac{\vartheta}{0.7}\right),\left(\frac{\eta}{0.3}, \frac{\vartheta}{0.8}\right),\left(\frac{\eta}{0.3}, \frac{\vartheta}{0.3}\right)>, G=<x,\left(\frac{\eta}{0.7}, \frac{j}{0.1}\right),\left(\frac{\eta}{0.3}, \frac{j}{0.2}\right),\left(\frac{\eta}{0.6}, \frac{\vartheta}{0.7}\right)>, H=<x,\left(\frac{\eta}{0.8}, \frac{\vartheta}{0.7}\right),\left(\frac{\eta}{0.9}, \frac{\vartheta}{0.8}\right),\left(\frac{\eta}{0.3}, \frac{\vartheta}{0.3}\right)>$.Define $I=<x,\left(\frac{\eta}{0.4}, \frac{\vartheta}{0.3}\right),\left(\frac{\eta}{0.3}, \frac{\vartheta}{0.8}\right),\left(\frac{\eta}{0.3}, \frac{\vartheta}{0.4}\right)$. Then $\operatorname{Nint}(I)=<x,\left(\frac{\eta}{0.2}, \frac{\vartheta}{0.3}\right),\left(\frac{\eta}{0.1}, \frac{\vartheta}{0.2}\right),\left(\frac{\eta}{0.7}, \frac{\vartheta}{0.7}\right)>$ also $N c l^{*} \operatorname{Nint}(I)=<x,\left(\frac{\eta}{0.7}, \frac{\vartheta}{0.7}\right),\left(\frac{\eta}{0.3}, \frac{\vartheta}{0.8}\right),\left(\frac{\eta}{0.3}, \frac{\vartheta}{0.3}\right)>$.It $\quad$ can $\quad$ be $\quad$ verified $\quad$ that $I \subseteq N^{*} c l(\operatorname{Nint}(I))$. So $I$ isneutrosophicsemi open*nevertheless not neutrosophic open.

Remark 2.9.(i) In any neutrosophic space $\left(\varkappa, \mathfrak{J}_{N}\right)$, it can be verified that neutrosophicsemi open*sets are $0_{N}$ and $1_{N}$.

Theorem 2.10.If $\left\{A_{\alpha}\right\}$ is a collection of neutrosophicsemi open*sets, then the arbitrary union $\cup A_{\alpha}$ is also neutrosophicsemi open*.

Proof: Since $A_{\alpha}$ is neutrosophicsemi open*for each $\alpha$, a neutrosophic open set $U_{\alpha}$ in $\mathcal{H}$ such that $U_{\alpha} \subseteq A_{\alpha} \subseteq N c l *\left(U_{\alpha}\right)$.Then $\cup U_{\alpha} \subseteq \cup A_{\alpha} \subseteq \cup N c l *\left(U_{\alpha}\right) \subseteq N c l *\left(\cup U_{\alpha}\right)$. Since $\cup U_{\alpha}$ is neutrosophic open, $\cup A_{\alpha}$ is Neutrosophicsemi open*.

Remark 2.11.It can be verified that the finite intersection of neutrosophicsemi open*sets need not be neutrosophicsemi open*.

The following example reveals the above fact.

Example 2.12.Let $\mathcal{X}=\{p, q\}, \quad \mathfrak{I}_{N}=\left\{0_{N}, G_{1}, G_{2}, 1_{N}\right\}$ be aneutrosophic topology on $\mathcal{H}$, where $G_{1}=<x,\left(\frac{p}{0.3}, \frac{q}{0.2}\right),\left(\frac{p}{0.5}, \frac{q}{0.4}\right),\left(\frac{p}{0.7}, \frac{q}{0.6}\right)>$ and $G_{2}=<x,\left(\frac{p}{0.2}, \frac{q}{0.1}\right),\left(\frac{p}{0.5}, \frac{q}{0.4}\right),\left(\frac{p}{0.4}, \frac{q}{0.3}\right)>$. Then neutrosophic subset A $=<x,\left(\frac{p}{0.3}, \frac{q}{0.2}\right),\left(\frac{p}{0.4}, \frac{q}{0.3}\right),\left(\frac{p}{0.7}, \frac{q}{0.8}\right)>$ and

B $\quad=<x,\left(\frac{p}{0.2}, \frac{\sigma}{0.2}\right),\left(\frac{\rho}{0.4}, \frac{q}{0.4}\right),\left(\frac{p}{0.8}, \frac{q}{0.6}\right)>\quad$ are $\quad$ neutrosophicsemi $\quad$ open*sets ${ }^{2}$ but A $\wedge B=<<x,\left(\frac{p}{0.2}, \frac{q}{0.2}\right),\left(\frac{p}{0.4}, \frac{q}{0.3}\right),\left(\frac{p}{0.8}, \frac{q}{0.8}\right)>$ is not neutrosophicsemi open*

But a neutrosophicsemi open* ${ }^{*}$ set intersect with a neutrosophic open set is neutrosophicsemi open*as shown in Theorem 2.14.

Theorem 2.13. If $G$ is neutrosophic open and $N c l^{*}(G \cap H) \subseteq N c l^{*}\left(G \cap N c l^{*}(H)\right)$ and hence $N c l^{*}(G) \cap H \subseteq N c l^{*}(G \cap$ $H$ ), where $H$ is aneutrosophic subset of a neutrosophic topological space.

Theorem 2.14.Aneutrosophic semi open* set intersect with a neutrosophic open set is neutrosophic semi open*.

Proof: Let $G$ beneutrosophicsemi open* and $H$ be a neutrosophic open set. Thenby definition,neutrosophic open set $U$ such that $U \subseteq G \subseteq N c l^{*}(U)$. Since $H$ is neutrosophic open, then $U \cap H \subseteq G \cap H \subseteq N c l^{*}(U) \cap H \subseteq N c l^{*}(U \cap H)$. Hence $G \cap H$ is neutrosophicsemi open*. 
Example 2.15.Let $\mathcal{H}=\{\varsigma, \xi\}, \quad \widetilde{I}_{N}=\left\{\begin{array}{lllllll}0, & G_{1}, & G_{2}, & 1\end{array}\right\}$ be aneutrosophic topology on $\mathcal{K}$, where $G_{1}$ $=<x,\left(\frac{\varsigma}{0.3}, \frac{\xi}{0.2}\right),\left(\frac{\varsigma}{0.5}, \frac{\xi}{0.4}\right),\left(\frac{\varsigma}{0.7}, \frac{\xi}{0.6}\right)>$ and $G_{2}=<x,\left(\frac{\varsigma}{0.2}, \frac{\xi}{0.1}\right),\left(\frac{\varsigma}{0.4}, \frac{\xi}{0.3}\right),\left(\frac{\varsigma}{0.8}, \frac{\xi}{0.7}\right)>$. Then neutrosophic $\quad$ subset $G=<x,\left(\frac{\varsigma}{0.3}, \frac{\xi}{0.2}\right),\left(\frac{\varsigma}{0.4}, \frac{\xi}{0.3}\right),\left(\frac{\varsigma}{0.7}, \frac{\xi}{0.8}\right)>$ is $\quad$ neutrosophicsemi $\quad$ open*set $\quad$ and $H=<x,\left(\frac{\varsigma}{0.2}, \frac{\xi}{0.1}\right),\left(\frac{\varsigma}{0.4}, \frac{\xi}{0.3}\right),\left(\frac{\varsigma}{0.8}, \frac{\xi}{0.7}\right)>$ is neutrosophic open but $\mathrm{G} \wedge H=<x,\left(\frac{\varsigma}{0.2}, \frac{\xi}{0.2}\right),\left(\frac{\varsigma}{0.4}, \frac{\xi}{0.3}\right),\left(\frac{\varsigma}{0.8}, \frac{\xi}{0.8}\right)>$ is not neutrosophicsemi open*.

Remark2.16.The collection of neutrosophicsemi open*sets [denoted by $N S-O *(X, \tau)$ ] forms a neutrosophic topology on $\varkappa$ if and only if it is closed under finite intersection.

Proposition 2.17.Union ofneutrosophicsemi open* $G$ and a neutrosophic open, $G \cup U$ is neutrosophicsemi open*.

Proof: Follows from Theorem 2.7 and Theorem 2.10.

Definition 2.18. For a neutrosophic topological space $\left(\mathcal{\varkappa}, \mathfrak{I}_{N}\right)$,let $\tau_{N S^{*}}=\left\{U \in N S-O *(\mathcal{K}, \mathfrak{I}): U \cap A \in N S-O *\left(\mathcal{K}, \mathfrak{I}_{N}\right)\right.$ for all $\left.A \in N S-O *\left(\mathcal{H}, \Im_{N}\right)\right\}$.

Theorem 2.19. $\tau_{N S^{*}}$ is a neutrosophic topology finer than $\left(\varkappa, \mathfrak{I}_{N}\right)$.

Proof: Clearly $0_{N}, 1_{N} \in \mathfrak{I}_{N S^{*}}$. Let $U_{\alpha} \in \mathfrak{I}_{N S^{*}}$ and $U=\cup U_{\alpha}$. Since $U_{\alpha} \in N S-O^{*}\left(\mathcal{X}, \mathfrak{I}_{N}\right)$, by using Theorem $2.10, U \in N S$ $O *\left(\mathcal{X}, \mathfrak{I}_{N}\right)$. Let $A \in N S-O *\left(\mathcal{K}, \mathfrak{I}_{N}\right)$. Then $U_{\alpha} \cap A \in N S-O *\left(\mathcal{X}, \mathfrak{I}_{N}\right)$, for each $\alpha$ and hence by Theorem $2.10, U \cap A=(\mathrm{U}$ $\left.U_{\alpha}\right) \cap A=\cup\left(U_{\alpha} \cap A\right) \in N S-O *\left(\mathcal{X}, \mathfrak{I}_{N}\right)$.Therefore $U \in \mathfrak{I}_{N S^{*}}$. Now let $U_{1}, U_{2}, \ldots, U_{n} \in \mathfrak{I}_{N S^{*}}$. Then $U_{1}, U_{2}, \ldots, U_{n} \in N S$ $O *\left(X, \mathfrak{I}_{N}\right)$ and by definition of $\mathfrak{I}_{N S^{*}}$, we get $\bigcap_{k=1}^{n} U_{k} \in N S-O *\left(\mathcal{X}, \mathfrak{I}_{N}\right)$. If $A \in N S-O *\left(X, \mathfrak{\Im}_{N}\right)$, then by repeated application of the condition, we have $\left(\bigcap_{k=1}^{n} U_{k}\right) \cap A \in N S-O *\left(X, \mathfrak{I}_{N}\right)$. Hence $A \in \mathfrak{I}_{N S^{*}}$. This shows that $\mathfrak{I}_{N S^{*}}$ is a neutrosophic topology on $X$.

Let $V \in \mathfrak{I}$. By using Theorem 2.7, $V \in N S-O *\left(\mathcal{X}, \mathfrak{I}_{N}\right)$. Also by Theorem $2.14, V \cap A \in N S-O *\left(\mathcal{X}, \mathfrak{I}_{N}\right)$ for all $A \in N S-O *\left(\varkappa, \Im_{N}\right)$. Hence $A \in \tau_{N S^{*}}$. Thus $\tau_{N S^{*}}$ is finer than $\left(\varkappa, \mathfrak{\Im}_{N}\right)$.

Theorem 2.20.Neutrosophicsemi open*set is neutrosophicsemi open.

Proof: Let $G$ be a neutrosophicsemi open*set. Then there is a neutrosophic open set $U$ in $X$ such that $U \subseteq G \subseteq N c l *(U)$. Note that $N c l *(U) \subseteq N c l(U)$. Therefore $U \subseteq G \subseteq N c l(U)$. Hence $G$ is neutrosophicsemi open.

The reverse of the above is not true.

Example 2.21.Let $\mathcal{X}=\{x\}$ and $\mathfrak{I}=\left\{0_{N}, 1_{N}, G, H, I, J\right\}$ where $G=\left\langle x, \frac{\mu}{0.5}, \frac{\gamma}{0.2}, \frac{\sigma}{0.6}\right\rangle, H=\left\langle x, \frac{\mu}{0.7}, \frac{\gamma}{0.4}, \frac{\sigma}{0.8}\right\rangle, I=<$ $x, \frac{\mu}{0.7}, \frac{\gamma}{0.4}, \frac{\sigma}{0.6}>, J=\left\langle x, \frac{\mu}{0.5}, \frac{\gamma}{0.2}, \frac{\sigma}{0.8}>\right.$. The corresponding neutrosophic closed sets are $\mathfrak{J}^{c}=\left\{0_{N}, 1_{N},<x, \frac{\mu}{0.6}, \frac{\gamma}{0.8}, \frac{\sigma}{0.5}>,<\right.$ $\left.x, \frac{\mu}{0.8}, \frac{\gamma}{0.6}, \frac{\sigma}{0.7}>,<x, \frac{\mu}{0.6}, \frac{\gamma}{0.6}, \frac{\sigma}{0.7}>,<x, \frac{\mu}{0.8}, \frac{\gamma}{0.8}, \frac{\sigma}{0.5}>\right\}$. Let $\mathrm{N}=<x, \frac{\mu}{0.5}, \frac{\gamma}{0.2}, \frac{\sigma}{0.8}>$. ThenNint $(\mathrm{\aleph})=\left\langle x, \frac{\mu}{0.5}, \frac{\gamma}{0.2}, \frac{\sigma}{0.8}>\right.$ follows that $N c l^{*}(\operatorname{Nint}(\mathcal{\aleph}))=\left\langle x, \frac{\mu}{0.4}, \frac{\gamma}{0.2}, \frac{\sigma}{0.6}>\right.$.Thusא $\nsubseteq N c l^{*}(\operatorname{Nint}(\boldsymbol{\aleph}))$ implies $\aleph$ is neutrosophicsemi open*. And it can be verified that $\aleph<x, \frac{\mu}{0.5}, \frac{\gamma}{0.2}, \frac{\sigma}{0.8}>\subseteq \operatorname{Ncl}(\operatorname{Nint}(\aleph))=\left\langle x, \frac{\mu}{0.8}, \frac{\gamma}{0.8}, \frac{\sigma}{0.5}>\right.$.It can be seen that $\boldsymbol{\aleph}$ is neutrosophicsemi open*set.

Theorem 2.22. If $L$ is neutrosophicsemi open*theniff $N c l *(L)=N c l *(\operatorname{Nint}(L))$.

Proof: By definition, $L \subseteq N c l^{*}(\operatorname{Nint}(L))$. Hence $N c l^{*}(L) \subseteq N c l^{*}(\operatorname{Nint}(L))$. Also we have $N c l^{*}(\operatorname{Nint}(L)) \subseteq N c l^{*}(L)$. Hence $N c l^{*}(\operatorname{Nint}(L))=N c l^{*}(L)$.

Conversely, Take $M=\operatorname{Nint}(L)$. Then $M$ is neutrosophic open set in $X$ such that $M \subseteq L \subseteq$ 
$N c l^{*}(\mathrm{~L})=N c l^{*}(\operatorname{Nint}(L))=N c l^{*}(L)$. Then by Definition 2.4, is neutrosophicsemi open*.

Theorem 2.23. Let $M$ be neutrosophicsemi open*and $N \subseteq X$ such that $M \subseteq N \subseteq N c l^{*}(M)$. Then $N$ is neutrosophicsemi open*.

Proof: Since $M$ is neutrosophicsemi open*, $M \subseteq N c l^{*}(\operatorname{Nint}(M))$. Since $\operatorname{Nint}(M) \subseteq N \operatorname{Nint}(N), N c l^{*}(N \operatorname{int}(M)) \subseteq$ $N c l^{*}(N \operatorname{Nint}(N))$. Therefore by the above theorem, $N \subseteq N c l^{*}(M)=N c l^{*}(N \operatorname{Nint}(M)) \subseteq N c l^{*}(N i n t(N))$. Hence by Definition 2.4, $N$ is neutrosophicsemi open*.

\section{NEUTROSOPHICSEMI CLOSED* SET}

Definition 3.1.Neutrosophic semi closed* setis known as the complement of a neutrosophic semi open*.

The neutrosophic generalized interior operator is symbolized by Nint*.

Definition 3.2. A neutrosophic semiregular*is known asboth neutrosophicsemi open*and neutrosophicsemi closed*.

Theorem 3.3. A neutrosophicset $\mathcal{M}$ is neutrosophicsemi closed $*$ iff $\operatorname{Nint} *(\mathcal{F}) \subseteq \mathcal{M} \subseteq \mathcal{F}$, where $\mathcal{F}$ is a neutrosophic closed set.

Proof: Suppose $\mathcal{M}$ is neutrosophicsemi closed*. Then by Definition $3.1, \mathcal{M}^{C}$ is neutrosophicsemi open*. Then by Definition 2.4, there existsneutrosophic open set $\mathcal{F}$ in $\varkappa$ such that $\mathcal{F} \subseteq \mathcal{M}^{C} \subseteq N c l^{*}(\mathcal{F})$ which implies $\mathcal{F}^{C} \supseteq \mathcal{M} \supseteq\left(N c l^{*}(F)\right)^{\mathrm{C}}$. $\operatorname{Since}\left(N c l^{*}(\mathcal{F})\right)^{C}=N i n t^{*}\left(\mathcal{M}^{C}\right), \mathcal{F}^{C} \supseteq \mathcal{M} \supseteq N i n t^{*}\left(\mathcal{F}^{C}\right)$ where $\mathcal{F}^{C}$ is neutrosophic closed in $\varkappa$.

Suppose a neutrosophic closed set $\mathcal{F}$ satisfying $N$ int $*(\mathcal{F}) \subseteq \mathcal{M} \subseteq \mathcal{F}$ which implies $\left(N i n t^{*}(\mathcal{F})\right)^{\mathrm{C}} \supseteq \mathcal{M}^{C} \supseteq \mathcal{F}^{C}$. Since $(\operatorname{Nint} *(\mathcal{F}))^{\mathrm{C}}=\operatorname{Ncl} l^{*}\left(\mathcal{F}^{C}\right)$, we have $N c l^{*}\left(\mathcal{F}^{C}\right) \supseteq \mathcal{M}^{C} \supseteq \mathcal{F}^{C}$ where $\mathcal{F}^{C}$ is a neutrosophic open set. Hence by Definition $2.1, \mathcal{M}^{C}$ is neutrosophicsemi open*. Hence the result.

Theorem 3.4.The Characterization of neutrosophicsemi closed*. That is, $A$ is neutrosophicsemi closed*iffNint* $(\operatorname{Ncl}(A)) \subseteq A$.

Proof: By Theorem $2.5, A^{C} \subseteq N c l^{*}\left(\operatorname{Nint}\left(A^{C}\right)\right.$. Taking complement on both sides and using the properties, $N i n t^{*}(N c l(A))$ $\subseteq A$.

Conversely, assume that $\operatorname{Nint} *(N \operatorname{cl}(A)) \subseteq A$. Take $\mathcal{M}=\operatorname{Ncl}(A)$. Then a neutrosophic closed set $\mathcal{M}$ in $X$ such that $\operatorname{Nint}^{*}(\mathcal{M}) \subseteq A \subseteq \mathcal{M}$ and hence $A$ is neutrosophicsemi closed*.

Theorem 3.5.Neutrosophic closed set is neutrosophicsemi closed*.

Proof:If $\mathbb{N}$ is Neutrosophic closed set. Then $\mathbb{N}^{C}$ is neutrosophic open. By Theorem 3.7, $\mathbb{N}^{C}$ is neutrosophicsemi open*. Hence $\mathbb{N}$ is neutrosophicsemi closed*.

Example 3.6.Let $\mathcal{H}=\{g, \hbar, \varepsilon\}$ and $\mathfrak{J}=\left\{0_{N}, A, B, C, D, 1_{N}\right\}$. Then $(\mathcal{x}, \tau)$ is a neutrosophictopological space. The neutrosophic closed sets are $\mathfrak{J}^{C}=\left\{1_{N}, E, F, G, H, 0_{N}\right\}$ where

$$
\begin{aligned}
& A=\left\langle x,\left(\frac{g}{0}, \frac{\hbar}{0.2}, \frac{\varepsilon}{0.9}\right),\left(\frac{g}{0.1}, \frac{\hbar}{0.8}, \frac{\varepsilon}{0.4}\right),\left(\frac{g}{0.5}, \frac{\hbar}{0.4}, \frac{\varepsilon}{0.3}\right)>\right. \\
& B=\left\langle x,\left(\frac{g}{0.4}, \frac{\hbar}{0.3}, \frac{\varepsilon}{0.3}\right),\left(\frac{g}{0.3}, \frac{\hbar}{0.7}, \frac{\varepsilon}{0.2}\right),\left(\frac{g}{0.3}, \frac{\hbar}{0.1}, \frac{\varepsilon}{0.7}\right)>,\right.
\end{aligned}
$$




$$
\begin{aligned}
& C=\left\langle x,\left(\frac{g}{0.4}, \frac{\hbar}{0.3}, \frac{\varepsilon}{0.9}\right),\left(\frac{g}{0.3}, \frac{\hbar}{0.8}, \frac{\varepsilon}{0.4}\right),\left(\frac{g}{0.3}, \frac{\hbar}{0.1}, \frac{\varepsilon}{0.3}\right)>,\right. \\
& D=\left\langle x,\left(\frac{g}{0}, \frac{\hbar}{0.2}, \frac{\varepsilon}{09}\right),\left(\frac{g}{0.1}, \frac{\hbar}{0.7}, \frac{\varepsilon}{0.2}\right),\left(\frac{g}{0.5}, \frac{\hbar}{0.4}, \frac{\varepsilon}{0.7}\right)>\right. \\
& E=\left\langle x,\left(\frac{g}{1}, \frac{\hbar}{0.8}, \frac{\varepsilon}{0.1}\right),\left(\frac{g}{0.9}, \frac{\hbar}{0.2}, \frac{\varepsilon}{0.6}\right),\left(\frac{g}{0.5}, \frac{\hbar}{0.6}, \frac{\varepsilon}{0.7}\right)>,\right. \\
& F=\left\langle x,\left(\frac{g}{0.6}, \frac{\hbar}{0.7}, \frac{\varepsilon}{0.7}\right),\left(\frac{g}{0.7}, \frac{\hbar}{0.3}, \frac{\varepsilon}{0.8}\right),\left(\frac{g}{0.7}, \frac{\hbar}{0.9}, \frac{\varepsilon}{0.3}\right)>,\right. \\
& G=\left\langle x,\left(\frac{g}{0.6}, \frac{\hbar}{0.7}, \frac{\varepsilon}{0.1}\right),\left(\frac{g}{0.7}, \frac{\hbar}{0.2}, \frac{\varepsilon}{0.6}\right),\left(\frac{g}{0.7}, \frac{\hbar}{0.9}, \frac{\varepsilon}{0.7}\right)>,\right. \\
& H=\left\langle x,\left(\frac{g}{1}, \frac{\hbar}{0.8}, \frac{\varepsilon}{0.1}\right),\left(\frac{g}{0.9}, \frac{\hbar}{0.3}, \frac{\varepsilon}{0.8}\right),\left(\frac{g}{0.5}, \frac{\hbar}{0.6}, \frac{\varepsilon}{0.3}\right)>\right.
\end{aligned}
$$

Define $I=<x,\left(\frac{g}{0.5}, \frac{\hbar}{0.6}, \frac{\varepsilon}{0.5}\right),\left(\frac{g}{0.5}, \frac{\hbar}{0.2}, \frac{\varepsilon}{0.4}\right),\left(\frac{g}{0.8}, \frac{\hbar}{1}, \frac{\varepsilon}{0.3}\right)>$.

Then $\operatorname{Ncl}(I)=<x,\left(\frac{g}{0.6}, \frac{\hbar}{0.7}, \frac{\varepsilon}{0.7}\right),\left(\frac{g}{0.7}, \frac{\hbar}{0.3}, \frac{\varepsilon}{0.8}\right),\left(\frac{g}{0.7}, \frac{\hbar}{0.9}, \frac{\varepsilon}{0.3}\right)><$ also it can be verified that $\operatorname{Nint}^{*}(\operatorname{Ncl}(I))=0_{N} \subseteq I$. Thus $I$ is neutrosophicsemi closed* but not neutrosophic closed.

Remark 3.7. We have seen that $0_{N}$ and $1_{N}$ are alwaysneutrosophicsemi closed* sets.

Theorem 3.8.If collection of neutrosophicsemi closed* sets $\left\{F_{\alpha}\right\}$, then arbitrary intersection, $\cap F_{\alpha}$ is also neutrosophicsemi closed*.

Proof: Since $\operatorname{each} F_{\alpha}$ is neutrosophicsemi $\operatorname{closed}^{*}, F_{\alpha}{ }^{C}$ is neutrosophicsemi open*. By Theorem 2.10 , $U F_{\alpha}{ }^{C}$ is neutrosophicsemi open*. That implies $\left(\cap F_{\alpha}\right)^{C}$ is neutrosophicsemi open*. Hence by Definition $3.1, \cap F_{\alpha}$ is neutrosophicsemi closed*.

Remark 3.9. We have found the result that finite union of two neutrosophicsemi closed* sets need not be neutrosophicsemi closed* as seen from the following examples.

Example 3.10.Let $\mathcal{H}=\{\kappa, \varrho\}$. Then the neutrosophic topology $\mathfrak{J}=\left\{\begin{array}{lllll}0_{N}, G_{1}, & G_{2}, & 1_{N}\end{array}\right\}$ where $G_{1}$ $=<x,\left(\frac{\kappa}{0.3}, \frac{\varrho}{0.2}\right),\left(\frac{\kappa}{0.5}, \frac{\varrho}{0.4}\right),\left(\frac{\kappa}{0.7}, \frac{\varrho}{0.6}\right)>$ and

$G_{2}=<x,\left(\frac{\kappa}{0.2}, \frac{\varrho}{0.1}\right),\left(\frac{\kappa}{0.4}, \frac{\varrho}{0.3}\right),\left(\frac{\kappa}{0.5}, \frac{\varrho}{0.4}\right)>$. The corresponding neutrosophic closed sets are $=\left\{0_{N}, G_{2}, G_{3}, 1_{N}\right.$ \} where $G_{3}=<x,\left(\frac{\kappa}{0.7}, \frac{\varrho}{0.8}\right),\left(\frac{\kappa}{0.5}, \frac{\varrho}{0.6}\right),\left(\frac{\kappa}{0.3}, \frac{\varrho}{0.4}\right)>$ and

$$
G_{4}=<x,\left(\frac{\kappa}{0.8}, \frac{\varrho}{0.9}\right),\left(\frac{\kappa}{0.6}, \frac{\varrho}{0.7}\right),\left(\frac{\kappa}{0.5}, \frac{\varrho}{0.6}\right)>
$$

Define neutrosophic subsets $G_{5}=<x,\left(\frac{\kappa}{0.3}, \frac{\varrho}{0.2}\right),\left(\frac{\kappa}{0.4}, \frac{\varrho}{0.3}\right),\left(\frac{\kappa}{0.4}, \frac{\varrho}{0.5}\right)>$ and

$$
\begin{aligned}
& G_{6}=<x,\left(\frac{\kappa}{0.2}, \frac{\varrho}{0.2}\right),\left(\frac{\kappa}{0.4}, \frac{\varrho}{0.4}\right),\left(\frac{\kappa}{0.4}, \frac{\varrho}{0.5}\right)>\text {. Then it can be found that } \\
& \operatorname{Ncl}\left(G_{5}\right)=<x,\left(\frac{\kappa}{0.7}, \frac{\varrho}{0.8}\right),\left(\frac{\kappa}{0.5}, \frac{\varrho}{0.6}\right),\left(\frac{\kappa}{0.3}, \frac{\varrho}{0.4}\right)>\text { and } \\
& \operatorname{Ncl}\left(G_{6}\right)=<x,\left(\frac{\kappa}{0.7}, \frac{\varrho}{0.8}\right),\left(\frac{\kappa}{0.5}, \frac{\varrho}{0.6}\right),\left(\frac{\kappa}{0.3}, \frac{\varrho}{0.4}\right)>,
\end{aligned}
$$


$\operatorname{Nint} *\left(N \operatorname{cl}\left(G_{5}\right)\right)=<x,\left(\frac{\kappa}{0.2}, \frac{\varrho}{0.1}\right),\left(\frac{\kappa}{0.4}, \frac{\varrho}{0.3}\right),\left(\frac{\kappa}{0.5}, \frac{\varrho}{0.4}\right)>\subseteq G_{5}$ and

$\operatorname{Nint} *\left(N \operatorname{Ncl}\left(G_{6}\right)\right)=<x,\left(\frac{\kappa}{0.2}, \frac{\varrho}{0.1}\right),\left(\frac{\kappa}{0.4}, \frac{\varrho}{0.3}\right),\left(\frac{\kappa}{0.5}, \frac{\varrho}{0.4}\right)>\subseteq G_{6}$, hence $G_{5}$ and $G_{6}$ are neutrosophic semi closed* sets but $G_{5} \vee G_{6}=<x,\left(\frac{\kappa}{0.3}, \frac{\varrho}{0.2}\right),\left(\frac{\kappa}{0.4}, \frac{\varrho}{0.4}\right),\left(\frac{\kappa}{0.4}, \frac{\varrho}{0.4}\right)>\quad$ is not neutrosophicsemi closed*.For, $N c l\left(G_{5} \vee G_{6}\right) \quad=$ $<x,\left(\frac{\kappa}{0.7}, \frac{\varrho}{0.8}\right),\left(\frac{\kappa}{0.5}, \frac{\varrho}{0.6}\right),\left(\frac{\kappa}{0.3}, \frac{\varrho}{0.4}\right)>, \operatorname{Nint} *\left(N \operatorname{cl}\left(G_{5} \vee G_{6}\right)\right)=<x,\left(\frac{\kappa}{0.3}, \frac{\varrho}{0.2}\right),\left(\frac{\kappa}{0.5}, \frac{\varrho}{0.4}\right),\left(\frac{\kappa}{0.7}, \frac{\varrho}{0.6}\right)>\nsubseteq G_{5} \vee G_{6}$.

But the union of a neutrosophicsemi closed* set and a neutrosophic closed set is neutrosophicsemi closed* as shown in Theorem 3.11 .

Theorem 3.11.Union ofneutrosophicsemi closed* and neutrosophic closed is neutrosophicsemi closed*.

Proof: Let us takeFbeneutrosophicsemi closed*. Then $F^{C}$ is neutrosophicsemi open*. Also we take G is neutrosophic closed, $G^{C}$ isneutrosophic open. By Theorem 2.14, $F^{C} \cap G^{C}=(F \cup G)^{C}$ is neutrosophicsemi open*. Finally $F \cup G$ is neutrosophicsemi closed*.

Corollary 3.12. Finite intersection of neutrosophicsemi closed* and neutrosophic closed is neutrosophicsemi closed*.

Proof: We take $F$ is neutrosophic closed and $E$ is neutrosophic closed, by Theorem 3.5, $F$ is neutrosophicsemi closed*. Then by Theorem 3.11, $E \cap F$ is neutrosophicsemi closed*.

Theorem 3.13. Eachneutrosophicsemi closed* set is neutrosophicsemi closed.

Proof: Let $F$ be a neutrosophicsemi closed* set. Then $F^{C}$ is neutrosophicsemi open*set. By Theorem $2.20, F^{C}$ is neutrosophicsemi open. Hence $F$ is neutrosophicsemi closed.

But each neutrosophic closed set may not be neutrosophic semi closed* as shown in the following examples.

Example 3.14.Let $\mathcal{X}=\{a\}$ and $\mathfrak{I}=\left\{0_{N}, 1_{N}, U, V, W, X\right\}$ where $U=\left\langle a, \frac{\mu}{0.5}, \frac{\gamma}{0.2}, \frac{\sigma}{0.6}\right\rangle$,

$V=<a, \frac{\mu}{0.7}, \frac{\gamma}{0.4}, \frac{\sigma}{0.8}>, W=<a, \frac{\mu}{0.7}, \frac{\gamma}{0.4}, \frac{\sigma}{0.6}>, X=<a, \frac{\mu}{0.5}, \frac{\gamma}{0.2}, \frac{\sigma}{0.8}>$.

The corresponding closed sets are $\mathfrak{J}^{c}=\left\{0_{N}, 1_{N}, Y, Z, S, T\right\}$ where $Y=<a, \frac{\mu}{0.5}, \frac{\gamma}{0.8}, \frac{\sigma}{0.4}>$, $Z=\left\langle a, \frac{\mu}{0.3}, \frac{\gamma}{0.6}, \frac{\sigma}{0.2}>, S=\left\langle a, \frac{\mu}{0.3}, \frac{\gamma}{0.6}, \frac{\sigma}{0.4}>, T=\left\langle a, \frac{\mu}{0.5}, \frac{\gamma}{0.8}, \frac{\sigma}{0.2}>\right.\right.\right.$.

Let $O=<a, \frac{\mu}{0.5}, \frac{\gamma}{0.8}, \frac{\sigma}{0.2}>$. Then it can be seen that $N c l(O)=\left\langle a, \frac{\mu}{0.5}, \frac{\gamma}{0.8}, \frac{\sigma}{0.2}\right\rangle$.and $\operatorname{Nint} *(N c l(O))=<$ $a, \frac{\mu}{0.5}, \frac{\gamma}{0.2}, \frac{\sigma}{0.4}>\nsubseteq<a, \frac{\mu}{0.5}, \frac{\gamma}{0.8}, \frac{\sigma}{0.2}>$. But

$\left.\operatorname{Nint}(\operatorname{Ncl}(O))=\left\langle a, \frac{\mu}{0.5}, \frac{\gamma}{0.8}, \frac{\sigma}{0.2}\right\rangle \subseteq<a, \frac{\mu}{0.5}, \frac{\gamma}{0.8}, \frac{\sigma}{0.2}\right\rangle$. Therefore $O$ is neutrosophicsemi closed but not neutrosophicsemi closed*.

Theorem 3.15.Let $A$ be neutrosophicsemi closed* and $B$ be a neutrosophic subset such that $N i n t^{*}(A) \subseteq B \subseteq A$.Then $B$ is neutrosophicsemi closed*.

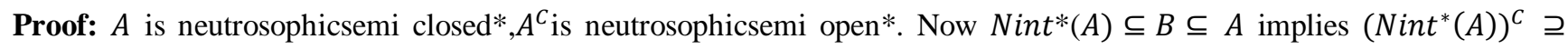
$B^{C} \supseteq A^{C}$. That is $\operatorname{Ncl}^{*}(A) \supseteq B^{C} \supseteq A^{C}$. Therefore byDefinition $2.4, B^{C}$ is neutrosophicsemi open*. Hence $B$ is neutrosophicsemi closed*. 
Theorem 3.16. Ais neutrosophicsemi closed*if and only ifNint $*(A)=\operatorname{Nint} *(\operatorname{Ncl}(A))$.

Proof: Since $A$ is neutrosophicsemi closed*, $N i n t^{*}(\operatorname{Ncl}(A)) \subseteq A$. Hence $N i n t *(N \operatorname{cl}(A)) \subseteq(N i n t *(A))$. Also we haveNint* $(A) \subseteq N i n t *(N \operatorname{Nl}(A))$. FinallyNint* $(N \operatorname{Nl}(A))=\operatorname{Nint}^{*}(A)$.

Conversely, Take $U=\operatorname{Ncl}(A)$. Thenneutrosophic closed set $U$ in $\varkappa$ such that $\operatorname{Nint} *(A)=\operatorname{Nint} *(\operatorname{Ncl}(A))=$ $N \operatorname{Nint}^{*}(A) \subseteq A \subseteq U$. Then by Theorem3.3, Ais neutrosophicsemi open*.

\section{CONCLUSIONS}

In this article, we had shown that the ideas of neutrosophicsemi open*and neutrosophicsemi closed* sets and its properties. Further, we have exhibited that the collection of neutrosophicsemi open*(closed*)sets lies in the middle of the collection of neutrosophicsemi open(closed) sets and the collection of neutrosophic open(closed) sets. In addition, some of their characterizations and fundamental properties of neutrosophicsemi open*(closed*) sets are also discussed.

\section{REFERENCES}

1. Arokiarani, R. Dhavaseelan, S. Jafari, M. Parimala: On Some New Notions and Functionsin Neutrosophic Topological Spaces, Neutrosophic Sets and Systems, Vol. 16 (2017), pp.16-19. doi.org/10.5281/zenodo.831915.

2. Atanassov, K. Intuitionistic fuzzy sets. In VII ITKR's Session; Publishing House: Sofia, Bulgaria, 1983.

3. Chang, C.L. Fuzzy topological spaces. J. Math. Anal. Appl.1968, 24, 182-190.

4. Coker, D. An introduction to intuitionistic fuzzy topological space, Fuzzy Sets Syst. 1997,88, 81-89.

5. Dhavaseelan, R.; Jafari, S. Generalized neutrosophic closed sets. In New Trends inneutrosophic Theory and Application; Smarandache, F., Pramanik, S., Eds.; Pons Editions: Brussels, Belgium., 2018, Volume 2, pp. 261-274.

6. P.Iswarya, and K.Bageerathi., On neutrosophic semi open sets in neutrosophic topological spaces, International Jour. Of Math. Trends and Tech.,214-223,(2016).

7. Jayanthi, D. Generalized closed sets in neutrosophic topological spaces. Int. J. Math.Trends Technol. Spec. Issue 2018, 10, 88-91.

8. Kavitha, C., TS Frank Gladson, and CM Arun Kumar. "Analysis Of Fuzzy Soft Set Compositions for Allocating Jobs to Machines in Fuzzy Environment."International Journal of Mechanical and Production Engineering Research and Development (IJMPERD) 8.1, Feb 2018, 229-242

9. Khairandish, Mohammad Omid, Ruchika Gupta, and Meenakshi Sharma. "A Hybrid Model of Faster R-Cnn and Svm for Tumor Detection and Classification of Mri Brain Images. "International Journal of Mechanical and Production Engineering Research and Development (IJMPERD)10.3, Jun 2020, 6863-6876

10. Mohapatra, Hitesh, et al. "A comparative analysis of clustering protocols of wireless sensor network." International journal of mechanical and production engineering research and development (IJMPERD) ISSN (P) (2020): 2249-6890.

11. D.Jayanthi, aGeneralized closed Sets in neutrosophic Topological Spaces, InternationalJournal of Mathematics Trends and Technology (IJMTT)- Special Issue ICRMIT March (2018).

12. Robert, A. and Pious Missier, S., A New Class of Nearly Open Sets, InternationalJournal ofMathematical Archive., 3(7), 2012, $1-8$. 
13. Salama, A.A.; Broumi, S.; Alblowi, S.A. Introduction to neutrosophic topological spatialregion, possible application to gis topological rules. Inf. Eng. Electron. Bus.2014, 6, 15-21.

14. Salama, A. A., and H. Elagamy. "Neutrosophic filters." International Journal of Computer Science Engineering and Information Technology Reseearch (IJCSEITR) 3.1 (2013): 307-312.

15. Salama, A.; Alblowi, S. Generalized neutrosophic set and Generalized NeutrousophicTopological Spaces, J. Comput. Sci. Eng. 2012, 2, 29-32.

16. Salama A. A and Alblowi S. A, Neutrosophic set and neutrosophic topological space, ISOR J. mathematics 3(4), 2012 , pp 31 35

17. Salama, A.A.; Smarandache, F.; Kroumov, V. neutrosophic closed set and neutrosophic continuousfunctions. Neutrosophic Sets Syst.2014, 4, 4-8.

18. Hanafy, I. M., A. A. Salama, and K. M. Mahfouz. "Neutrosophic classical events and its probability." International Journal of Mathematics and Computer Applications Research (IJMCAR) Vol.(3) 1 (2013): 171-178.

19. Smarandache. F :, neutrosophic Set: - A Generalization of Intuitionistic Fuzzy set,Journal of Defense Resources Management1,107-114,(2010).

20. V. Venkateswara Rao., Y. Srinivasa Rao., neutrosophic Pre-open Sets and Pre-closedSets in neutrosophic Topology, International Journal of ChemTech Research, Vol.(10),No.10, pp 449-458, (2017).

21. Zadeh, L. Fuzzy sets. Inform Control 1965, 8, 338-353. 
